\title{
The SideWinder for powering a hand-coring auger in drilling and lifting
}

\author{
Jay KYNE, ${ }^{1}$ Joe McCONNELL ${ }^{2}$ \\ ${ }^{1}$ Ice Coring and Drilling Services, Space Science and Engineering Center, University of Wisconsin - Madison, \\ 1225 West Dayton Street, Madison, WI 53706-1612, USA \\ E-mail: jayk@ssec.wisc.edu \\ ${ }^{2}$ Desert Research Institute, 2215 Raggio Parkway, Reno, NV 89512-1095, USA
}

\begin{abstract}
The SideWinder is a power delivery system for a hand-coring ice auger. It not only greatly increases the rate of drilling, it also makes it possible for a single operator to drill to depths difficult and cumbersome for hand-coring systems. The SideWinder power is delivered by an electric drill that is used for both the drilling and the lifting of the drill string. A high-strength low-stretch synthetic-fiber rope lifts and lowers the drill string as it winds around a tube attached to the electric drill. During winding, the synthetic-fiber rope is guided onto the winding tube by rope cleats that also serve as a quick way to secure the rope for drilling. The end of the winding tube connects directly to the protruding extension of the drill string for drilling. The rope always remains attached to the drill string by a rope-pin that replaces the bottommost connecting pin; the winding tube holds the rope and always stays attached to the electric drill. For lifting and lowering the drill string, cradle arms on a frame that hinges on a plywood platform hold the winding tube.
\end{abstract}

\section{INTRODUCTION}

A 'hand' ice-coring auger is so called because it is operated manually, with human muscle providing both the power to turn the auger and to lift the drill stem in and out of the borehole. These systems are designed with torsion stem pieces (extensions) that pin together to extend the drill string from the surface to the borehole bottom, and a T-handle or a crank for turning the drill string by hand. The torsion stem turns a simple coring barrel that collects the core and ice chips within the barrel. The turning of the drill string by hand is very slow and tedious. Beyond depths of about $6 \mathrm{~m}$, the drill string becomes heavy and cumbersome for one person to lift from the borehole, especially in windy conditions. Drilling manually beyond about $6 \mathrm{~m}$ depth involves breaking the drill string into more manageable lengths, which requires some way to suspend the down-hole portion while disconnecting and handling the stem above. This introduces the risk of dropping the down-hole partial string and barrel back down the borehole. Also, as the firn becomes denser with depth, turning the drill string by hand becomes an ever more tedious, arduous and time-consuming task.

\section{DEVELOPMENT}

Early attempts at powering a hand-coring system involved accessorizing a Polar Ice Coring Office (PICO) hand-coring auger with electrical power for drilling and a tripod for lifting (Koci, 1984). This adaptation yielded only limited success. The tripod with a pulley system for mechanical advantage had a limited lifting length and required collars to hold and lift the drill string when tripping in and out of the borehole. This proved to be time-consuming and cumbersome.

The SideWinder adaptation enables one person to core to greater depths than are usually practical, even for two or three people, when drilling by hand, by providing power for the turning and lifting stages of drilling (Figs 1 and 2). Additionally, it eliminates the risk of dropping the string back down the hole since a rope tether is always attached at the top of the core barrel. Continually raising the drill string out of the hole with the SideWinder, one person can easily and quickly break the drill stem into manageable lengths of $3-5 \mathrm{~m}$ by engaging a hand brake on the winder once the desired amount of stem protrudes from the borehole, leaving both hands free to unpin and remove the desired length of stem. The hand brake is a bent rod that simply hooks a cleat preventing reverse winding. The hand brake falls away by gravity when the winding begins again. Going back into the hole is just the reverse of coming out: the driller stops the lowering of the stem, sets the hand brake, adds the next stem section, and disengages the hand brake by giving the drill handle a quick pull downward that allows the brake to fall from the cleat.

The SideWinder was first used in 1997 in Greenland and in successive seasons, taking cores in over 60 locations with an average depth of about $20 \mathrm{~m}$. During field deployments the authors flew to preselected sites and, with the plane waiting, were able to drill, process and pack a $20 \mathrm{~m}$ core, often in under 3 hours from landing to take-off, using this system. Subsequently, depths of up to $30 \mathrm{~m}$ have been achieved in Greenland and Antarctica. Although 40 or $50 \mathrm{~m}$ depths are achievable in principle with the SideWinder, drilling beyond a depth of about $30 \mathrm{~m}$ is better addressed with a different system. Beyond this depth the excessive weight of the drill stem, consequent surface handling difficulties, and long trip times in and out of the borehole become rate-limiting factors. The SideWinder system is currently available for checkout by field parties through the Antarctic support contractor in McMurdo Station.

\section{METHOD AND USE}

After arriving at the drilling site, set-up takes about $10 \mathrm{~min}$. The plywood drilling platform is placed on a level area of the snow surface. A hole in the platform exposes the snow for drilling. All the SideWinder components, except for the 


\section{The SideWinder accessory \\ to a \\ hand core augering system}

\author{
A reversible electric \\ $3 / 4$ inch drill with a universal \\ motor uses DC or AC current \\ controlled by a variable \\ transformer to obtain the correct \\ rpm for drilling.
}

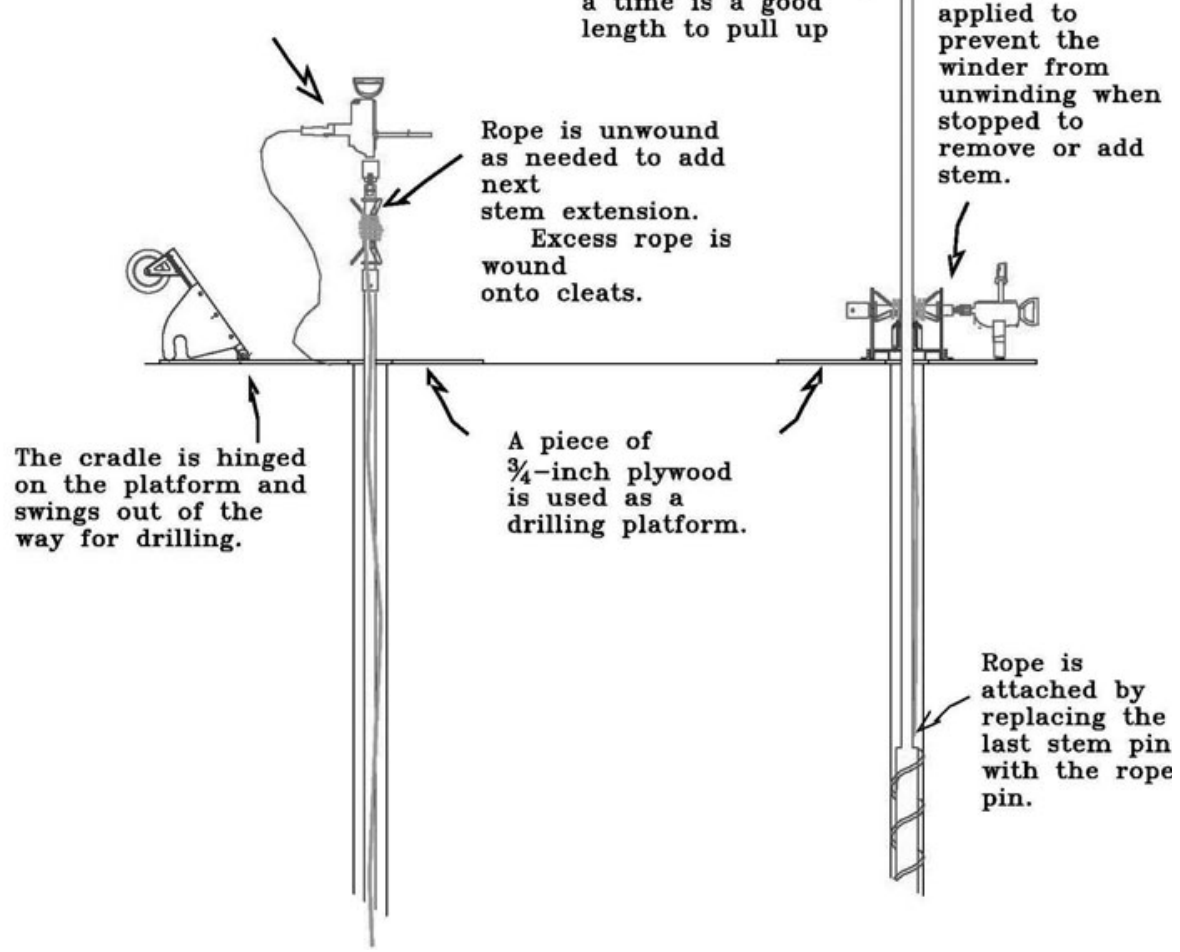

Fig. 1. The front cover of the SideWinder User's Manual showing its use.

generator and the plywood platform, come in one box. The cradle frame needs no assembly and quickly pins to hinges on the plywood. The winder tube is chucked into the electric drill where it remains for the duration of the drilling.

The first core is taken using only the simple auger barrel, turned by hand and using the horizon as a guide for vertical orientation. This core is nearly the length of the coring barrel, since the chips churning to the surface are simply brushed away. The next few cores are drilled with the electric drill rotating the drill string, but the lifting of the drill string is done by hand while it can be managed as a single piece. At a depth of 5 or $6 \mathrm{~m}$, when lifting the drill string out of the borehole as one piece becomes heavy and awkward, lifting it with the electric drill motor becomes more expedient. To use the electric drill for lifting the drill string, several meters of rope are unwound from the winder - enough rope to allow free movement of the barrel into an upright position over the hole without tugging on the winder/electric drill lying on the platform nearby. The end of the rope is then attached to the drive adapter (the piece between the barrel and the first torsion stem piece) by replacing that connecting pin with the rope pin, which is tied by a non-slipping loop to the end of the rope. The rope remains connected throughout the remainder of the core collection, that is the barrel adapter and the first extension remain pinned together. The barrel is then placed into the borehole and suspended by the rope held in one hand while a bar is placed through the rope loop at the rope pin with the other hand. The bar is laid onto the platform and suspends the barrel. This leaves the top of the barrel just below the surface and the end of the first extension protruding above the surface of the platform. With the bar suspending the barrel, both of the operator's hands are free to drop the cradle into place and position the winder within it. The excess rope is wound taut and the bar is removed. Next, a stem section is connected and then the drill string is lowered to the next point of attachment. The transition from lowering the drill string to the bottom to drilling is very simple. When the string reaches the bottom and the new extension has been added, the drill/winder is lifted from the cradle and the cradle is swung back out of the way. The electric drill/winder is then lifted and placed onto the protruding extension end and pinned in place. Loose rope is hooked onto the cleats and then drilling begins. The rope does not need to be taut for drilling.

When the drilling is finished, the core is broken from the bottom, a task the electric drill is not powerful enough to do. 


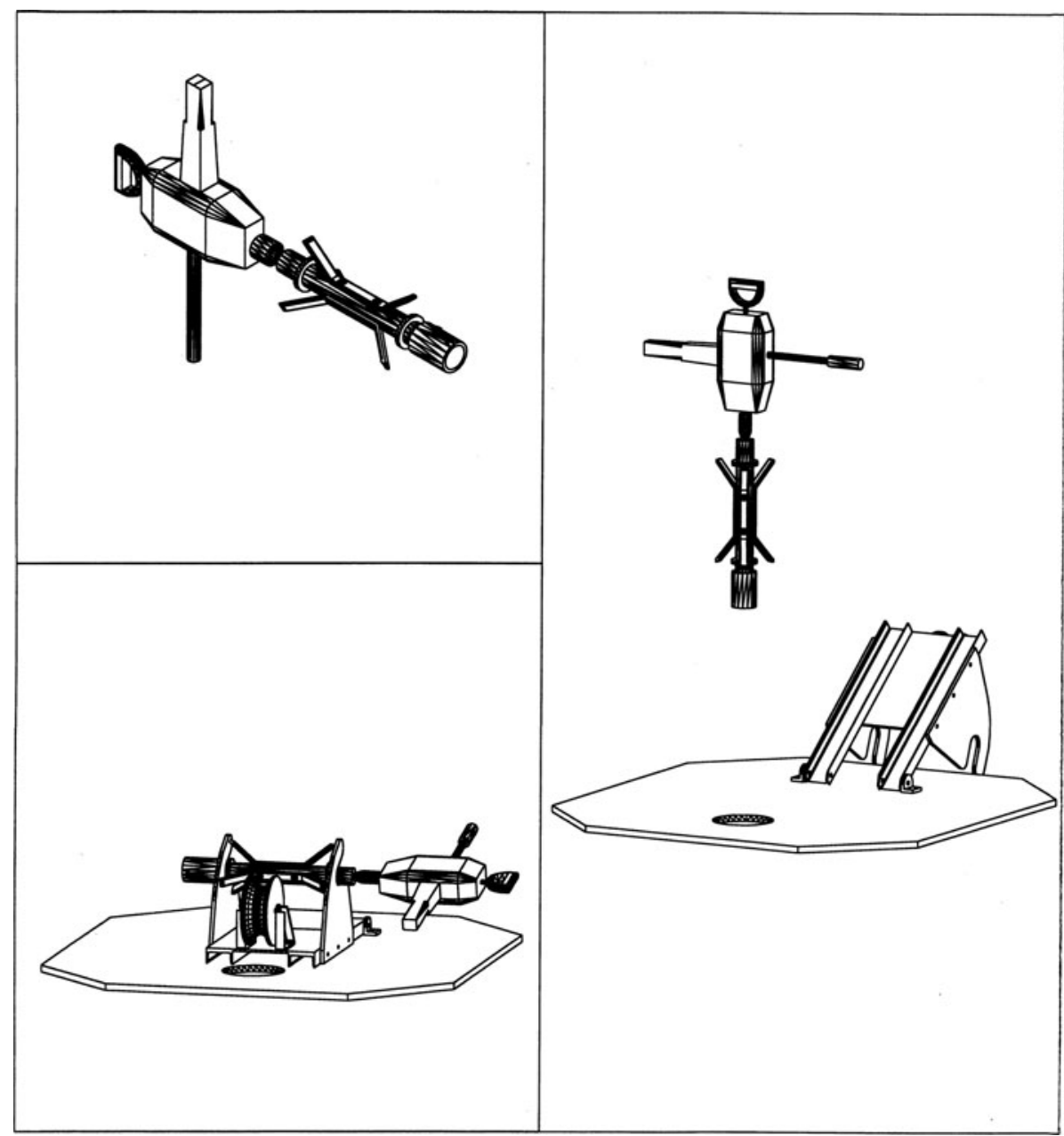

Fig. 2. Three-dimensional drawing showing the electric drill/winder and the SideWinder in its drilling and lifting positions.

Breaking is accomplished by lifting the drill string manually until the break is felt. This step is the most physically demanding aspect of the drilling due in part to the selfanchoring nature of chip-augering flights of the auger barrel, but primarily due to the tension needed to break the core.

Once the core is broken, the barrel is recovered by reversing the tripping-down process. The electric drill/ winder is unpinned and the cradle is swung back into the down position. The electric drill/winder is laid into the cradle and is ready immediately for lifting the entire drill string. The rope can be wound so that the pipe handle of the electric drill pushes against the plywood platform and carries the torque when it is running. The drill is then used to lift the string from the borehole, stopping periodically when the desired length of torsion stem has emerged from the borehole. When stopping to remove manageable lengths of stem, the operator simply releases the trigger on the electric drill handle and engages the catch brake. When all the extensions except the last one are removed in manageable lengths and the barrel is just below the surface, the bar is placed through the loop in the rope and tension is taken off the winder by releasing the lock lever. Enough rope is spooled out to permit lifting the barrel from the hole without interference from the rope. The electric drill/winder is removed from the cradle, placed nearby and the cradle is lifted to its back position. The barrel is then lifted from the hole and the core removed and processed.

\section{FURTHER DEVELOPMENT}

A second-generation SideWinder Lite utilizes a lighter and less powerful electric drill. This drill has a dial speed control on the handle and consequently does not require a variable transformer to control the speed of rotation. This adaptation also uses less power: a small $2 \mathrm{~kW}$ generator is adequate even at higher altitudes. This system should perform well with a 3 in $(\sim 7.6 \mathrm{~cm})$ coring barrel at least up to $20 \mathrm{~m}$. The weight saving of the SideWinder Lite over the SideWinder is about $20 \mathrm{lb}(9 \mathrm{~kg})$. The SideWinder can also be combined with the PrairieDog double-barreled corer for improved core recovery and more efficient drill operations (Kyne and McConnell, 2007).

\section{CARGO WEIGHTS}

Aside from the weight of the hand-auger system, the SideWinder has the following weights:

The SideWinder complete in its box: $105 \mathrm{lb}(48 \mathrm{~kg})$.

The $2 \mathrm{~kW}$ generator with box: $60 \mathrm{lb}(27 \mathrm{~kg}$ ) (based on the Honda 2000i, $47 \mathrm{lb}(21 \mathrm{~kg})$ ).

The plywood drilling platform: $40 \mathrm{lb}(18 \mathrm{~kg})$.

Gasoline: usage is about $0.1 \mathrm{gal}(0.38 \mathrm{~L})$ per kWh.

The SideWinder Lite has the following weight changes:

The SideWinder Lite complete in its box: $85 \mathrm{lb}(39 \mathrm{~kg})$. 


\section{DISCUSSION AND CONCLUSION}

Use of the SideWinder to power a hand-coring auger system works well for taking 10-30 m of core within a day. It offers possibilities for day trips by snowmobile, sled or small aircraft from a central base with minimal personnel. To achieve depths greater than $25-30 \mathrm{~m}$, a lightweight version of an electrical-mechanical 'tethered' drill is desirable since time per core meter and the weight of the stem increase to the point where a hand-coring system is no longer time- or weight-efficient. However, the cost of the SideWinder is a fraction of that of a tethered electromechanical drill and it offers an alternative to more logistically intensive heavier and larger systems.

\section{ACKNOWLEDGEMENTS}

The SideWinder was made using funds provided by both the NASA Program for Arctic Regional Climate Assessment (PARCA) and the US National Science Foundation (NSF) Office of Polar Programs.

\section{REFERENCES}

Koci, B.R. 1984. A lightweight handcoring auger. CRREL Spec. Rep., 84-34. 55-59.

Kyne, J. and J. McConnell. 2007. The Prairie Dog: a double-barrel coring drill for 'hand' augering. Ann. Glaciol., 47, 99-100. 